\title{
Health impact of acute intermittent porphyria in latent and non-recurrent attacks patients
}

\author{
Juan Buendía-Martínez ${ }^{1,3^{*}+}$, María Barreda-Sánchez ${ }^{2,3+}$, Lidya Rodríguez-Peña ${ }^{4}$, \\ María Juliana Ballesta-Martínez 2,3,4,5, Vanesa López-González 2,4,5,9, María José Sánchez-Soler 2,3,4,5, \\ Ana Teresa Serrano-Antón 4,9 María Elena Pérez-Tomás², Remedios Gil-Ferrer ${ }^{2,4}$, Francisco Avilés-Plaza ${ }^{6}$, \\ Guillermo Glover-López ${ }^{2,5,7}$, Carmen Carazo-Díaz ${ }^{8}$ and Encarna Guillén-Navarro 2,4,5,**i]
}

\begin{abstract}
Background: Acute intermittent porphyria (AIP) is a genetic disease characterized by acute neurovisceral attacks. Long-term clinical conditions, chronic symptoms and impaired health related quality of life (HRQoL) have been reported during non-attack periods but mainly in patients with recurrent attacks. Our aim was to investigate these aspects in sporadic AIP (SA-AIP) and latent AIP (L-AIP) patients. Fifty-five participants, 27 SA-AIP ( $<4$ attacks/year) and $28 \mathrm{~L}$-AIP patients with a prevalent founder mutation from Spain were included. Medical records were reviewed, and individual interviews, physical examinations, biochemical analyses, and abdominal ultrasound scans were conducted. HRQoL was assessed through an EQ-5D-5L questionnaire. A comparative study was made between SA-AIP and L-AIP patients.

Results: The earliest long-term clinical condition associated with SA-AIP was chronic kidney disease. Chronic symptoms were reported in $85.2 \%$ of SA-AIP and $46.4 \%$ of L-AIP patients. Unspecific abdominal pain, fatigue, muscle pain and insomnia were significantly more frequent in SA-AIP than in L-AIP patients. The EQ-5D-5L index was lower in SAAIP (0.809 vs. $0.926, p=0.0497)$, and the impact of "pain", "anxiety-depression" and "mobility" was more intense in the EQ-5D-5L domains in SA-AIP than in L-AIP subjects and the general Spanish population.

Conclusions: AIP remains a chronically symptomatic disease that adversely affects health and quality of life, even in patients with low rate of acute attacks. We suggest a regular monitoring of patients with symptomatic AIP regardless of their attack rate or the time since their last attack, with proper pain management and careful attention to kidney function.
\end{abstract}

Keywords: Acute intermittent porphyria, Chronic symptoms, Quality of life, Sporadic attacks, Chronic kidney disease

\footnotetext{
*Correspondence: juanbuendi@hotmail.com; guillen.encarna@gmail.com; encarna.guillen@carm.es

†Juan Buendía-Martínez and María Barreda-Sánchez contributed equally to this work as first authors

${ }^{1}$ Servicio de Neurología, Hospital General Universitario Morales Meseguer, Av Marqués de los Vélez, s/n, 30008 Murcia, Spain ${ }^{4}$ Sección Genética Médica, Servicio de Pediatría, Hospital Clínico Universitario Virgen de la Arrixaca (HCUVA), Ctra. Madrid-Cartagena s/n., CP 30120 El Palmar (Murcia), Spain

Full list of author information is available at the end of the article
}

\section{Background}

Acute intermittent porphyria (AIP, MIM\#176000) is the most common type of acute hepatic porphyrias (AHP) [1]. AIP is an autosomal dominant condition caused by a deficiency of hydroxymethylbilane synthase (HMBS; EC 2.5.1.61). Although most $H M B S$ pathogenic variants are private, founder effects have been reported [2]. Such is the case with the HMBS pathogenic variant NM_ original author(s) and the source, provide a link to the Creative Commons licence, and indicate if changes were made. The images or other third party material in this article are included in the article's Creative Commons licence, unless indicated otherwise in a credit line to the material. If material is not included in the article's Creative Commons licence and your intended use is not permitted by statutory regulation or exceeds the permitted use, you will need to obtain permission directly from the copyright holder. To view a copy of this licence, visit http://creativecommons.org/licenses/by/4.0/. The Creative Commons Public Domain Dedication waiver (http://creativeco mmons.org/publicdomain/zero/1.0/) applies to the data made available in this article, unless otherwise stated in a credit line to the data. 
000190.3(HMBS):c.669_698del30 p.(Glu223_Leu232) in the Region of Murcia, south-east Spain [3]. As a consequence, AIP prevalence has increased in this part of the country [4].

The clinical course of AIP is characterized by acute neurovisceral attacks involving the autonomic, peripheral and central nervous systems, with intervening non-attack periods $[5,6]$. During acute attacks, the level of porphyrin precursors, aminolevulinic acid (ALA) and porphobilinogen (PBG) are markedly increased. Elevated urine PBG (U-PBG) is the main biochemical diagnostic marker of AHP attack. After recovery from a neurovisceral attack, the levels of urinary ALA (U-ALA) and U-PBG may remain high for long periods of time [7-9].

Most individuals with HMBS deficiency are latent AIP (L-AIP) subjects as they never experience neurovisceral attacks. However, manifest AIP patients may suffer from sporadic $(<4$ per year) or recurrent $(>4$ per year) neurovisceral attacks [10].

The most frequent long-term AIP-related clinical conditions are hypertension (HT), chronic kidney disease (CKD), and hepatocellular carcinoma (HCC) [11-13]. Chronic symptoms, such as abdominal and muscle pain, weakness, nausea, tiredness, anxiety and insomnia, have been described in the periods between acute attacks [14-19]. In addition, a decrease in health-related quality of life (HRQoL) has been reported in AHP patients compared to the general population, even in periods between attacks [14-20]. However, studies on these issues are scarce and those that exist mainly target patients with recurrent attack AHP (RA-AHP). Therefore, the aim of this study was to assess AIP impact on the health status during non-attack periods in sporadic attack patients (SA-AIP), which is the most common form of symptomatic AIP, and L-AIP subjects, focusing on chronic symptoms associated to the disease, HRQoL and AIP long-term clinical conditions.

\section{Materials and methods Patients}

L-AIP subjects and SA-AIP patients from the Region of Murcia were included in this study after giving their written informed consent. All of them had a confirmed molecular AIP diagnosis, and were routinely followed in the Medical Genetics Section of the Hospital Clínico Universitario Virgen de la Arrixaca (HCUVA). This study was approved by the Ethical Committee of the HCUVA and all procedures were performed according to the Helsinki Declaration.

SA-AIP patients were defined by having had at least one previous neurovisceral attack and an annual rate of attacks lower than 4 per year. A neurovisceral attack was considered to have been suffered when the subject was admitted to hospital due to clinical compatible symptoms, with raised U-PBG levels, and received specific treatment. L-AIP status was defined when there was no neurovisceral attack background. Subjects were catalogued as hypertensive if they were under current antihypertensive treatment.

\section{Biochemical assessments}

Spot U-ALA and U-PBG were assessed by spectrophotometry after column chromatography (BioSystems S.A., Barcelona, Spain), and results were normalized to urinary creatinine (U-ALA/Cr and U-PBG/Cr).

Serum liver enzymes (AST, ALT and GGT), serum ferritin and blood haemoglobin were determined by validated assays in the HCUVA clinical laboratory.

\section{Long-term clinical conditions and chronic symptoms}

Clinical information was obtained by individual interview and a review of medical records. A self-made clinical questionnaire was used to identify chronic symptoms of interest. A physical examination and an abdominal ultrasound scan were conducted. CKD was considered with an estimated glomerular filtration rate (eGFR) lower than $60 \mathrm{~mL} / \mathrm{min}$ per $1.73 \mathrm{~m}^{2}$, or with kidney damage markers, or both, in a minimum period of 3 months [21]. The eGFR was calculated using the CKD-EPI formula.

\section{HRQoL assesment}

The EuroQoL 5-dimensions questionnaire 5-levels (EQ5D-5L) was used to assess HRQoL [22]. The EQ-5D-5L measures five dimensions: "mobility", "self-care", "usual activities", "pain/discomfort" and "anxiety/depression". The 5 dimension scores are converted into a single index score ranging from 0 to 1 (EQ-5D-5L index) with " 1 " meaning optimal health. EQ-5D-5L includes a self-rated health assessment using a visual analogue score (VAS), with "100" signifying maximum and best health. Normative data from EQ-5D-5L in the Spanish population was available for comparison [23].

\section{Statistical approach}

Continuous variables were summarized with means and standard deviations (SD), while qualitative variables were summarized with proportions. Multiple linear regression models were used to test associations between continuous variables and explanatory variables. Fisher's tests were used to test associations between qualitative variables. Multiple logistic regression models were used to study association between U-ALA/Cr and U-PBG/Cr (exposures) and the different outcomes were coded as binary categories. All these models were adjusted by sex and age. Data were analysed using $\mathrm{R}$ (3.4.1. version) software package. 


\section{Results}

Of the 55 individuals included with confirmed $H M B S$ pathogenic variants, $80 \%$ carried the founder mutation NM_000190.3: c.669_698del30 p.(Glu223_Leu232). Other pathogenic variants were NM_000190.3:c.76 C> T p.Arg26Cys (8 patients, 14.54\%), NM_000190.3:c.750delA p.(Glu250GlufsTer4) (2 patients, 3.64\%) and NM_000190.3:c.275T >C p.(Leu92Pro) (1 patient, 1.82\%). Thirty-two (58.2\%) were females. Mean age was 44.7 years (16-77): 45.7 years (2177 ) in women, and 43.9 years (16-75) in men. Results in SA-AIP and L-AIP patients are detailed in Table 1.

Twenty-seven patients $(49.1 \%)$ had a history of SAAIP and $28(50.9 \%)$ were L-AIP, with mean ages of 47.5 years (28-77) and 41.3 years (16-75) respectively. There was higher proportion of females in the SA-AIP group $(74.1 \%, 20 / 27)$ than in L-AIP $(42.8 \%, 12 / 28)$. The mean background number of neurovisceral attacks per patient was 2.27 (SD 1.51): 2.45 (1-6, SD 1.5) in women, and 1.66 $(1-3$, SD 0.98$)$ in men. Most SA-AIP patients $(81.5 \%$, $22 / 27$ ) had suffered only 3 or less attacks in their lifetime. $85.2 \%(23 / 27)$ had had no attacks in the year preceding this study (average number of attacks in this period was 0.15 per patient). No patient had suffered an attack in the last 6 months and the average time since the last attack was 16.17 years (SD 12.6).

There were no statistical differences in the number of background neurovisceral attacks between founder mutation carriers and carriers of the rest of the pathogenic variants.

\section{Long-term clinical conditions}

Main clinical conditions in SA-AIP and L-AIP are shown in Fig. 1. $14.5 \%(8 / 55)$ of AIP individuals had received a previous $\mathrm{CKD}$ diagnosis at the mean age of 34.6 years (19-55). All of them had SA-AIP (8/27), showing significant differences in the frequency of CKD compared to L-AIP $(p=0018)$. Accordingly, SA-AIP patients had a significantly lower eGFR than L-AIP subjects $(\mathrm{p}<0.0001)$ (Table 1). Proteinuria, if present, was below $200 \mathrm{mg} / \mathrm{g}$ creatinine (0-192) in all cases.

$30.9 \%$ of subjects $(17 / 55)$ were currently following antihypertensive treatment. There was no substantial difference in the prevalence of HT in SA-AIP and L-AIP (Fig. 1), nor in the age of onset (SA-AIP: $47.44 \pm 17.78$ years; L-AIP: $47.25 \pm 5.82$ years; $\mathrm{p}=0.9759)$ or the number of antihypertensive agents needed for the proper control (SA-AIP: $1.44 \pm 0.73$; L-AIP: $1.88 \pm 1.13$; $\mathrm{p}=0.3741$ ).

Serum liver enzymes were above normal levels in $11,1 \%(3 / 27)$ of SA-AIP and $3 \%(1 / 27)$ of L-AIP subjects, although the average levels of ALT, AST and GGT were within normal limits in both sets of patients (Table 1).

\begin{tabular}{|c|c|c|c|}
\hline & SA-AIP & L-AIP & $p$ value \\
\hline N (\%) & $27(49.1 \%)$ & $28(50.9 \%)$ & - \\
\hline Age $($ mean $\pm S D)$ & $47.5 \pm 15.6$ & $41.3 \pm 15.7$ & - \\
\hline Age range (years) & [28-77] & {$[16-75]$} & - \\
\hline Females, N (\%) & $20(74.1 \%)$ & $12(42.8 \%)$ & - \\
\hline Males, N (\%) & $7(25.9 \%)$ & $18(57.1 \%)$ & - \\
\hline c.669_698del30 carriers ${ }^{\mathrm{a}}$, N (\%) & $20(74.1 \%)$ & $24(85.7 \%)$ & - \\
\hline $\mathrm{BMI}($ mean $\pm \mathrm{SD})$ & $26.93 \pm 4.4$ & $28.17 \pm 4.93$ & 0.3436 \\
\hline \multicolumn{4}{|l|}{$\begin{array}{l}\text { Biochemical assessments } \\
\quad(\text { mean } \pm \text { SD) }\end{array}$} \\
\hline eGFR (ml/min per $\left.1.73 \mathrm{~m}^{2}\right)$ & $73.16 \pm 27.3$ & $105.07 \pm 19.2$ & $<0.0001^{*}$ \\
\hline AST (U/L) & $28.19 \pm 6.5$ & $22.31 \pm 8.6$ & $0.0082^{*}$ \\
\hline $\operatorname{ALT}(U / L)$ & $27.04 \pm 14.4$ & $25.3 \pm 10.8$ & 0.6119 \\
\hline GGT (U/L) & $20.24 \pm 18.5$ & $26.52 \pm 17.6$ & 0.3047 \\
\hline $\mathrm{Hb}$ in males $(\mathrm{g} / \mathrm{dl})$ & $13.54 \pm 1.73$ & $14.4 \pm 1.36$ & 0.0563 \\
\hline Ferritin in males (ng/ml) & $57.59 \pm 64.26$ & $80.46 \pm 65.08$ & 0.2373 \\
\hline $\mathrm{Hb}$ in females $(\mathrm{g} / \mathrm{dl})$ & $12.64 \pm 0.89$ & $13.34 \pm 0.95$ & 0.1270 \\
\hline Ferritin in females (ng/ml) & $53.44 \pm 37.5$ & $43.73 \pm 68.7$ & 0.6513 \\
\hline $\mathrm{U}-\mathrm{PBG} / \mathrm{Cr}^{\mathrm{b}}(\mu \mathrm{mol} / \mathrm{mmol})$ & $17.09 \pm 15.93$ & $1.32 \pm 2.29$ & $0.0001^{*}$ \\
\hline $\mathrm{U}-\mathrm{ALA} / \mathrm{Cr}^{\mathrm{b}}(\mu \mathrm{mol} / \mathrm{mmol})$ & $9.86 \pm 6.76$ & $6.06 \pm 4.35$ & 0.1333 \\
\hline \multicolumn{4}{|l|}{ HRQoL scores (mean $\pm \mathrm{SD}$ ) } \\
\hline EQ-5D-5L index & $0.809 \pm 0.16$ & $0.926 \pm 0.14$ & $0.0497^{*}$ \\
\hline EQ-5D-5L VAS & $75.44 \pm 16.16$ & $86.78 \pm 15.78$ & 0.1353 \\
\hline
\end{tabular}

SA-AIP sporadic attack AIP, L-AIP latent AIP, $N$ total number of participants, $B M I$ body mass index, eGFR estimated glomerular filtrate rate, AST amino aspartate transferase, $A L T$ alanine aminotransferase, GGT gamma glutamyl transferase, $\mathrm{H} b$ haemoglobin; ferritin; U-PBG/Cr urinary porphobilinogen/ creatinine ratio, $U-A L A / C r$ urinary aminolevulinic acid/creatinine ratio, VAS visual analogue scale

${ }^{*} P$ value $\leq 0.05$ adjusted by age and gender. Laboratory reference ranges: eGFR > 60mL/min/1.73m2; AST 5-35 U/L; ALT 10-40 U/L; GGT 7-50 U/L; Hb $12-16 \mathrm{~g} / \mathrm{dL}$; Ferritin $12-300 \mathrm{ng} / \mathrm{mL}$; U-PBG/Cr $<1.5 \mu \mathrm{mol} / \mathrm{mmol} ; \mathrm{U}-\mathrm{ALA} / \mathrm{Cr}<3.8$ $\mu \mathrm{mol} / \mathrm{mmol}$

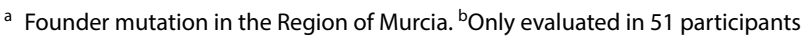

AST was significantly higher in SA-AIP patients than in L-AIP. No hepatic or other abdominal abnormalities were detected by ultrasound examination. Serum ferritin and haemoglobin levels were similar in the SA-AIP and L-AIP groups.

\section{Reported chronic symptoms}

Chronic symptoms were reported in $85.2 \%$ (23/27) of SA-AIP patients and $46.4 \%(13 / 28)$ of L-AIP. Recurrent non-specific abdominal pain followed by fatigue, muscle pain, anxiety and insomnia, were the most prevalent symptoms in SA-AIP with a significantly higher frequency than in L-AIP $(p=0.0023, p=0.0129, p=0.0261$, $\mathrm{p}=0.1022, \mathrm{p}=0.0437$, respectively), with the exception of anxiety which, followed by fatigue and muscle pain, were the most frequent symptoms in L-AIP (Fig. 1). 


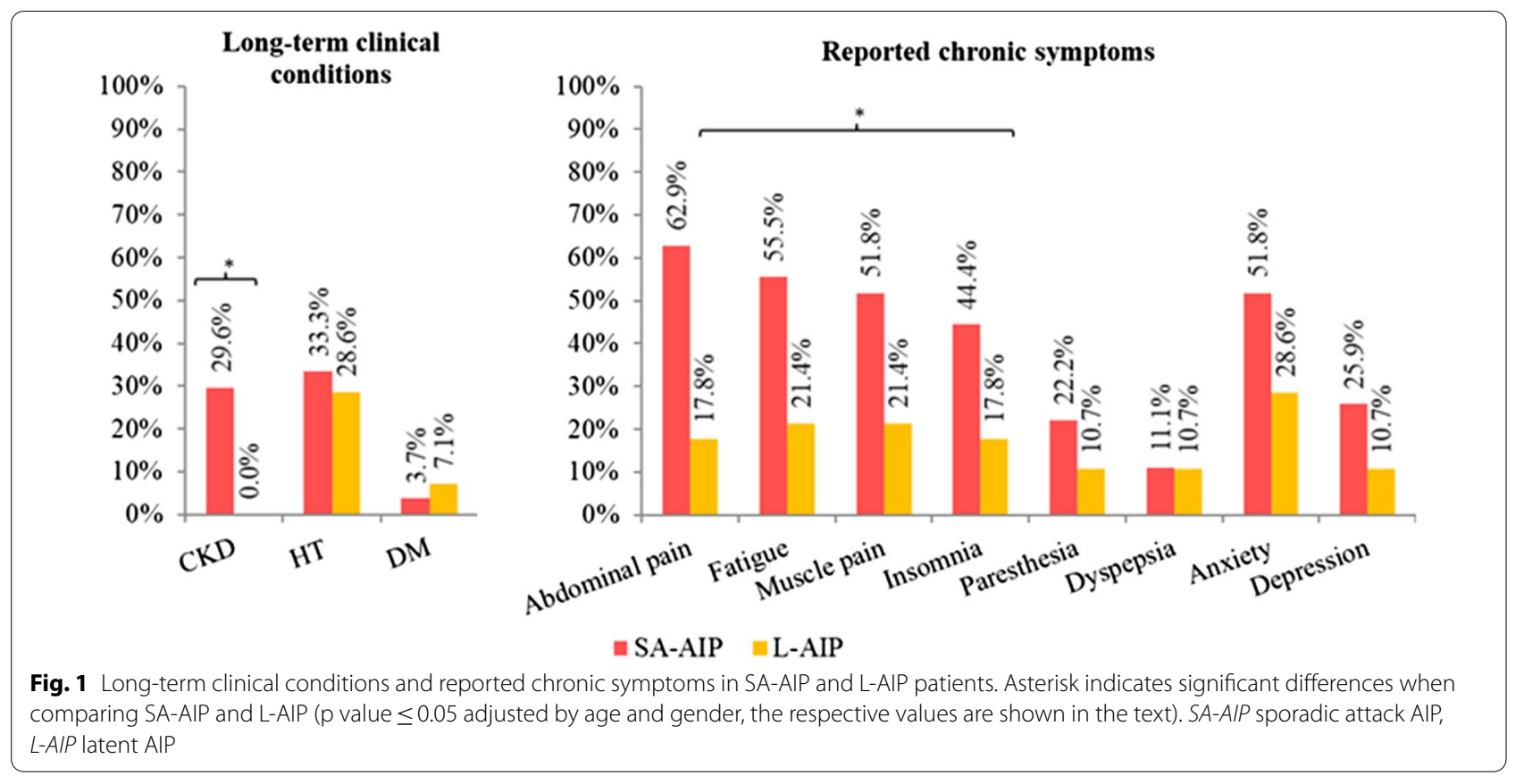

Anxiety was the main psychiatric problem in both groups, while depressive symptoms less frequent.

\section{HRQoL assessment}

EQ-5D-5L data analysis showed that "mobility", "pain/ discomfort" and "anxiety/depression" domains had significantly worse intensity in SA-AIP than L-AIP (Fig. 2).
Pain perception was greater in SA-AIP than in L-AIP $(\mathrm{p}=0.0001)$. Out of 27 SA-AIP patients, $23(85.2 \%)$ suffered pain to some degree, and $8(29.6 \%)$ to a moderate or severe degree (Fig. 2). In contrast, $32.2 \%(9 / 28)$ of L-AIP subjects reported pain, but only $7.2 \%$ to a "more than slight" degree. Anxiety/depression problems were also of greater intensity among SA-AIP patients $(\mathrm{p}=0.0201)$ since L-AIP subjects had only slight

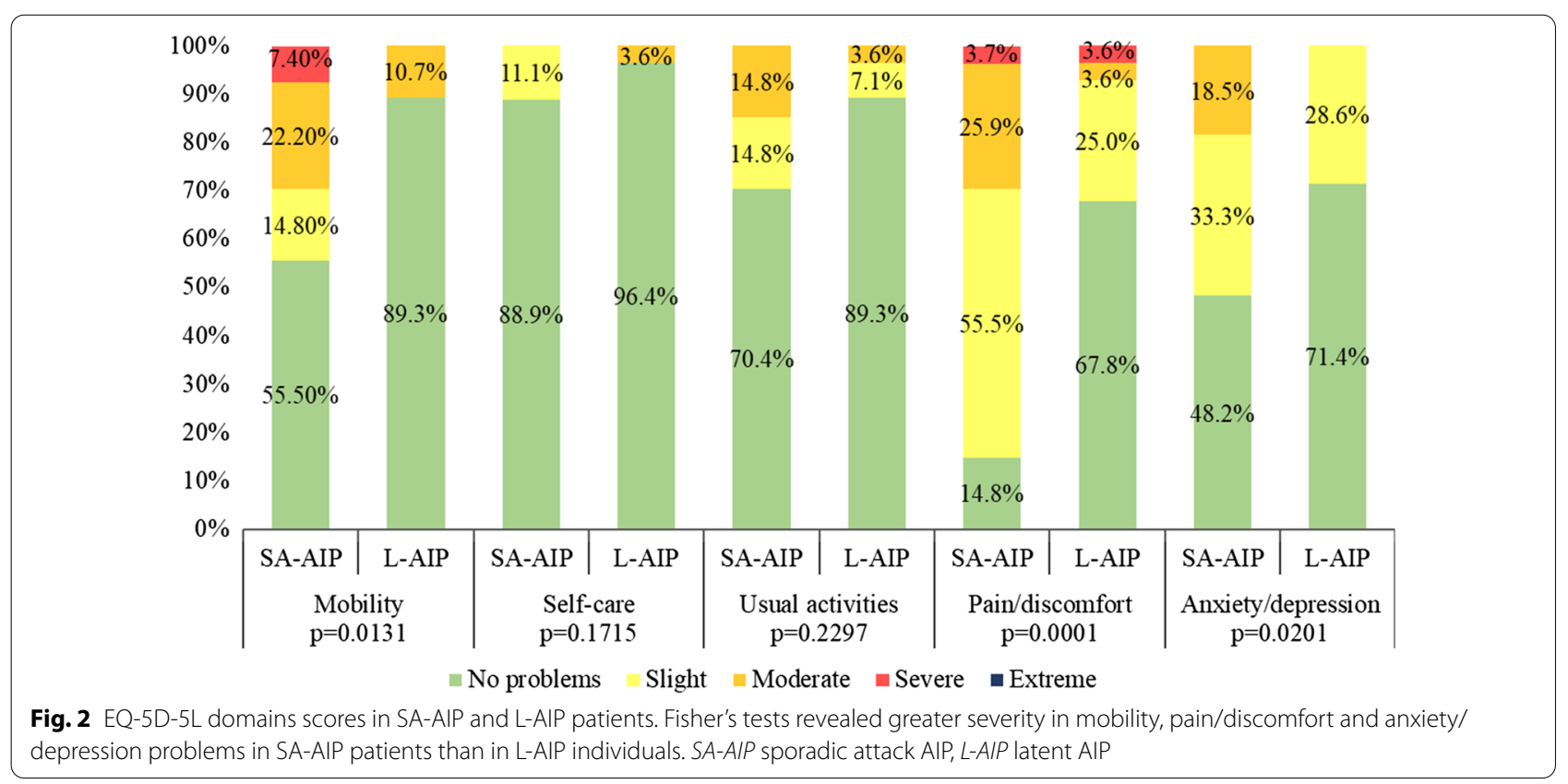


symptoms. Meanwhile, $18.5 \%(5 / 27)$ of SA-AIP patients reported moderate anxiety/depression problems. Finally, "mobility problems" were of greater severity in SA-AIP than in L-AIP $(\mathrm{p}=0.0131)$, with $44.4 \%(12 / 27)$ of SAAIP patients reporting mobility problems, $29.6 \%(8 / 27)$ to a moderate or severe degree. Analysis of the causes of impaired mobility showed that arthritis, previous stroke sequel or chemotherapy-induced peripheral neuropathy were some of the causes, but the main cause in SA-AIP patients with moderate or severe grade problems was a residual axonal polyneuropathy associated with a previous AIP acute attack $(5 / 8,62.5 \%)$.
The mean EQ-5D-5L index and VAS were on average worse in SA-AIP than in L-AIP (Table 1). When EQ5D-5L index was compared by percentiles with those in Spanish normative population by age ranges (Table 2), lower indexes in SA-AIP and minor differences with L-AIP were observed. The lowest EQ-5D-5L index in SAAIP outstand, especially in the 25-34 and the 35-44 age ranges, which are below the 10th percentile.

With regard to the domains included in the EQ-5D-5L questionnaire, Fig. 3 shows the proportion of patients who reported more than "slight problems" for each level, meaning that only levels "moderate problems," "severe

Table 2 Spanish normative population, SA-AIP and L-AIP EQ-5D-5L index values expressed by age ranges

\begin{tabular}{|c|c|c|c|c|c|c|c|}
\hline \multirow[t]{2}{*}{ Age range } & \multirow{2}{*}{$\begin{array}{l}\text { Spanish normative values } \\
\text { EQ-5D-5L index }\end{array}$} & \multicolumn{3}{|l|}{ SA-AIP } & \multicolumn{3}{|l|}{ L-AIP } \\
\hline & & EQ-5D-5L index & $\mathbf{N}$ & Percentile $^{\mathbf{b}}$ & EQ-5D-5L index & $\mathbf{N}$ & Percentile $^{b}$ \\
\hline $18-24$ & $0.983(0.07)$ & - & - & - & $1.00(0.00)$ & 5 & >P95 \\
\hline $25-34$ & $0.977(0.07)$ & $0.880(0.10)$ & 4 & P5-P10 & $0.970(0.05)$ & 6 & P50-P60 \\
\hline $35-44$ & $0.957(0.11)$ & $0.808(0.13)$ & 11 & P5-P10 & $0.943(0.08)$ & 5 & P20-P30 \\
\hline $45-54$ & $0.934(0.14)$ & $0.940(0.05)$ & 3 & P30-P40 & $0.971(0.06)$ & 5 & P50-P60 \\
\hline $55-64$ & $0.904(0.17)$ & $0.743(0.15)$ & 6 & P10-P20 & $0.796(0.25)$ & 4 & P10-P20 \\
\hline $65-74$ & $0.870(0.20)$ & $0.846(0.09)$ & 2 & P10-P20 & $0.811(0.22)$ & 3 & P20-P30 \\
\hline $75-84$ & $0.785(0.24)$ & 0.342 & 1 & P5-P10 & - & - & - \\
\hline
\end{tabular}

Values are reported as mean (SD)

$N$ total number of individuals, SA-AIP sporadic attack AIP, L-AIP latent AIP

a Spanish normative values for EQ-5D-5L index and percentiles were extracted from Hernandez, 2018 [23], bercentile of the EQ-5D-5L index with respect to the Spanish normative values

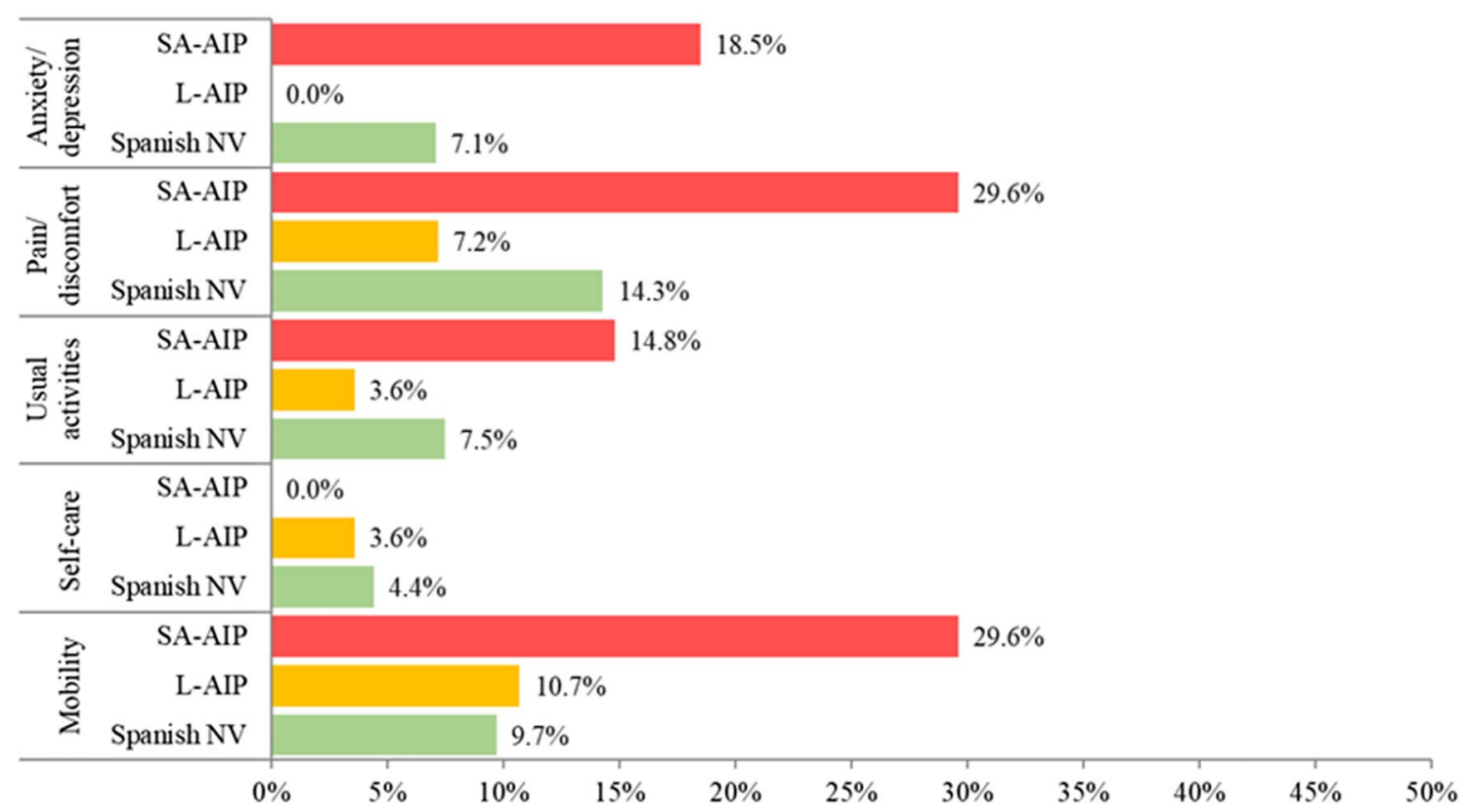

Fig. 3 Categorized score (including: moderate, severe or extreme scores) in EQ-5D-5L domains. Spanish NV Spanish normative population, L-AIP latent AIP, SA-AIP sporadic attack AIP 
problems," and "extreme/unable" were considered. The published results available in the Spanish normative population have also been included. There was a higher proportion of SA-AIP patients with "mobility", "pain/ discomfort", "anxiety/depression" and "usual activities" problems than L-AIP patients or individuals in the normative population. The latter two groups had similar proportions in the different domains, except for a slightly higher proportion of problems in the "pain/discomfort" and "usual activities" domains, observed in L-AIP.

\section{Urine ALA and PBG assessments}

Urine samples were collected from 51 individuals $(24$ SA-AIP and 27 L-AIP). The U-PBG/Cr, but not U-ALA/ $\mathrm{Cr}$, was significantly higher in SA-AIP than in L-AIP (Table 1). In SA-AIP patients, U-PBG/Cr values correlate with U-ALA/Cr levels: for each unit increase in U-ALA/ $\mathrm{Cr}$, there was an average increase of $2.06 \mathrm{U}-\mathrm{PBG} / \mathrm{Cr}$ units (CI 95\% 1.7-2.4, $\mathrm{p}<0.001)$. In L-AIP no significant association was found between U-ALA /Cr and U-PBG/ $\mathrm{Cr}$; and higher U-ALA/Cr levels did not correlate with higher levels of U-PBG/Cr, whose values remained similar among these patients (data not shown).

A regression study was carried out to assess a possible relationship between $\mathrm{U}-\mathrm{ALA} / \mathrm{Cr}$ and $\mathrm{U}-\mathrm{PBG} / \mathrm{Cr}$, on the one hand, and fatigue, muscle or abdominal pain and the level of anxiety and pain on the other (EQ-5D-5L data, classified as "absent or slight problems" and "more than slight problems"). No significant relationship was found between the levels of porphyrin precursors and the aforementioned chronic symptoms.

\section{Discussion}

To the best of our knowledge, this is the first study describing chronic manifestations in an AIP population with SA-AIP, which represents the majority of patients with symptomatic AIP, and L-AIP. The findings show that long-term clinical conditions and chronic symptoms more frequently occur in SA-AIP patients compared with L-AIP. Also, the quality of life of patients with SAAIP was seen to be more impaired compared with L-AIP patients.

As described by Pallet et al. [12], impaired kidney function and CKD was strongly associated with SA-AIP condition. This suggests that there may be an increased risk of CKD even in patients who have only had one or two acute attacks in their lives, as was the case in this study. This relation is reinforced by the fact that the age of CKD diagnosis and the age of first AIP attack were quite close. In addition, the prevalence of renal impairment in SAAIP resembles that seen in other AHP cohorts [19].

In contrast to other investigations [11, 12, 24, 25], HT prevalence was similar to that described in the Spanish reference population [26], and there was no difference between SA-AIP and L-AIP. Pallet et al. described HT as a frequent complication in AIP patients with symptoms [12], but the average age of their cohort was substantially higher (65 years) than ours.

To date, no cases of HCC have been diagnosed, and biochemical and ultrasound examination have not revealed any other liver abnormalities. It should be noted that the participants in this study had an average age of less than 50 years, the age above which an increased risk of HCC has been described [27, 28]. Longer-term followup and a larger cohort may be needed to appreciate the overall relationship of SA-AIP and L-AIP with HT, liver function and HCC risk.

The available published data had shown that HRQoL is impaired in AIP, and self-reported chronic symptoms range from 18 to $22 \%$ in patients with less severe AHP to $65-78 \%$ in patients with RA-AHP [15, 17-19]. Overall, we found that the frequency of chronic symptoms in SAAIP patients $(85.2 \%)$ was almost twice than that of L-AIP patients $(46.4 \%)$.

The differences in pain symptom frequencies between SA-AIP and L-AIP are noteworthy. Pain, mainly recurrent and non-specific abdominal pain and/or generalized muscle pain, should be considered as one of the main chronic symptoms associated with symptomatic AIP, as it was in the EQ-5D-5L questionnaire. Fatigue (excluding iron deficiency anaemia as a possible cause of fatigue) and insomnia were also more relevant problems in SAAIP than in L-AIP. Anxiety, which was also shown to be a common symptom, was not significantly more frequent in the SA-AIP than in the L-AIP, although it was more intense in the former on the basis of the results of the EQ-5D-5L questionnaire. In general, the symptoms in SA-AIP and their extent appear similar to those reported in RA-AHP [17-19].

EQ-5D-5L also revealed more mobility problems in SA-AIP patients than in L-AIP, which may have been due to AIP axonal polyneuropathy sequels in our SA-AIP group. AIP related acute neuropathy was no progressive and the patients who suffered from it tended to improve with slight sequels.

All of the above is consistent with worse health status reported in SA-AIP than in L-AIP patients, through the 5Q-5D-5L index and VAS. This suggests, as expected, that overall health status is related with the frequency of acute attacks and disease activity. Although HRQoL studies that include a healthy control group would be needed, here we also show evidence of a worse QoL reported by SA-AIP patients than that published in the Spanish normative population.

Finally, higher U-PBG/Cr levels were found in SA-AIP patients than in L-AIP patients, as already known [29]. 
This may be explained by increased disease activity in SA-AIP and because porphyrins precursors remain elevated for long periods of time after an acute attack. As has been reported before, U-PBG/Cr levels in SA-AIP patients were proportional to those of U-ALA/Cr [7]. Since U-PBG/Cr was not related to U-ALA/Cr in L-AIP (even in subjects with high U-ALA/Cr), this finding may indicate that apart from HBMS deficiency (present in L-AIP and SA-AIP), there is a sustained dysfunction in the haem metabolic pathway of patients with SAAIP. These data must be carefully interpreted and further research is needed to assess this hypothesis and its possible relationship with the development of chronic symptoms.

Since the average time without attacks in SA-AIP patients was more than 15 years, in our opinion our findings should not be attributed to possible sequels or manifestations associated with recent attacks, which could lead to a bias in the study. It is important to note that mean age in the SA-AIP sample was 47.5 years. This is still a relatively young population, and the full chronic impact of the disease may not have fully developed. Conversely, this means that the findings observed in this study may be considered as early-onset manifestations. This hypothesis is reinforced by the worse EQ-5D-5L index results in the young adult age ranges observed in SA-AIP compared to the normative population.

However, these findings should be corroborated by further studies in larger SA-AIP populations. In our opinion, it is worth extending the studies related with these aspects, which until now have been carried out mainly in patients with recurrent attacks, to patients who are subject to sporadic attacks.

\section{Conclusions}

In summary, the present study shows that AIP has a negative impact on health, evidencing that patients with SAAIP have significant chronic symptoms and long-term clinical conditions compared to L-AIP, despite low attack rates or long periods without attacks.

AIP should not be considered only as an acute disease, since neurovisceral attacks are not the only clinical expression of the disease.

This reinforces the need for regular monitoring of patients with symptomatic AIP regardless of their attack rate or the time since their last attack. Special attention should be paid to kidney function in order to detect early deterioration and progression to CKD.

\footnotetext{
Abbreviations

AHP: Acute hepatic porphyria; AIP: Acute intermittent porphyria; $\mathrm{Cr}$ : Creatinine; CKD: Chronic kidney disease; eGFR: Estimated glomerular filtration rate; EQ-5D-5L: EuroQoL 5-dimensions questionnaire 5-levels; HCC: Hepatocellular
}

carcinoma; HMBS: Hydroxymethylbilane synthase; HRQoL: Health related quality of life; HT: Hypertension; L-AIP: Latent acute intermittent porphyria; RA-AHP: Recurrent attack acute hepatic porphyria; SA-AIP: Acute intermittent porphyria; U-ALA: Urine aminolevulinic acid; U-PBG: Urine porphobilinogen; VAS: Visual analogue score.

\section{Acknowledgements}

We wish to acknowledge all the AIP patients and families for their generous participation in this study.

\section{Authors' contributions}

JB-M, MB-S and EG-N conceived and planned the study; MJB-M, VL-G, MJS-S, LR-P, ATS-A, MM-A, GG-L, FA-P and EG-N contributed to patient diagnosis, biochemical and molecular characterization; JB-M, MB-S, MJB-M, VL-G, MJS-S, ATS-A, LR-P and EG-N, contributed to patient recruitment and the acquisition of clinical data; JB-M, MB-S, MEP-T and RG-F contributed in sample collection; CC-D performed statistical analysis; JB-M, MB-S and EG-N contributed to the analysis of the results and wrote the manuscript; all authors provided critical feedback of analysis and helped shape the manuscript. All authors read and approved the final manuscript.

\section{Funding}

This research was partly founded by Universidad Católica de Murcia (UCAM) (Grant PMAFI/09/14).

\section{Availability of data and materials}

The datasets used and/or analysed during the current study are available from the corresponding author on reasonable request.

\section{Ethics approval and consent to participate}

This study was approved by the Ethical Committee of Clinical Investigation in Hospital Clínico Universitario Virgen de la Arrixaca and all procedures were performed according to the Helsinki Declaration. Written informed consent was obtained from all participants.

\section{Consent for publication}

Not applicable.

\section{Competing interests}

María Barreda-Sánchez and Encarna Guillén-Navarro received research grants and financial support for attending scientific meetings from Orphan Europe S.A.R.L and Alnylam Pharmaceuticals Inc. Encarna Guillén-Navarro has occasionally acted as a consultant for Alnylam Pharmaceutical Inc. The rest of the authors declare that they have no competing interests.

\section{Author details \\ 1 Servicio de Neurología, Hospital General Universitario Morales Meseguer, Av Marqués de los Vélez, s/n, 30008 Murcia, Spain. ${ }^{2}$ Instituto Murciano de Investigación Biosanitaria Virgen de la Arrixaca (IMIB-Arrixaca), Murcia, Spain. ${ }^{3}$ Facultad de Ciencias de la Salud, Universidad Católica de Murcia (UCAM), Murcia, Spain. ${ }^{4}$ Sección Genética Médica, Servicio de Pediatría, Hospital Clínico Universitario Virgen de la Arrixaca (HCUVA), Ctra. Madrid-Cartagena s/n., CP 30120 El Palmar (Murcia), Spain. ${ }^{5}$ CIBERER-ISCIII, Madrid, Spain. ${ }^{6}$ Servi- cio de Análisis Clínicos, HCUVA, Murcia, Spain. ${ }^{7}$ Centro de Bioquímica y Gené- tica Clínica, HCUVA, Murcia, Spain. ${ }^{8}$ Applied Statistical Methods in Medical Research Group, UCAM, Murcia, Spain. ${ }^{9}$ Departamento de Cirugía, Pediatría y Obstetricia y Ginecología, Facultad de Medicina, Universidad de Murcia (UMU), Murcia, Spain.}

Received: 20 October 2020 Accepted: 11 February 2021

Published online: 27 February 2021

\section{References}

1. Elder G, Harper P, Badminton M, Sandberg S, Deybach JC. The incidence of inherited porphyrias in Europe. J Inherit Metab Dis. 2013;36(5):849-57.

2. Andersson C, Floderus Y, Wikberg A, Lithner F. The W198X and R173W mutations in the porphobilinogen deaminase gene in acute intermittent porphyria have higher clinical penetrance than R167W. A populationbased study. Scand J Clin Lab Invest. 2000;60(7):643-8. 
3. Guillen-Navarro E, Carbonell P, Glover G, Sanchez-Solis M, FernandezBarreiro A. Novel HMBS founder mutation and significant intronic polymorphism in Spanish patients with acute intermittent porphyria. Ann Hum Genet. 2004;68:509-14.

4. Barreda-Sanchez M, Buendia-Martinez J, Glover-Lopez G, Carazo-Diaz C, Ballesta-Martinez MJ, Lopez-Gonzalez V, et al. High penetrance of acute intermittent porphyria in a Spanish founder mutation population and CYP2D6 genotype as a susceptibility factor. Orphanet J Rare Dis. 2019;14(1):59.

5. Puy H, Gouya L, Deybach JC. Porphyrias. Lancet. 2010;375(9718):924-37.

6. Bissell DM, Wang B. Acute hepatic porphyria. J Clin Transl Hepatol. 2015;3(1):17-26.

7. Kauppinen R, von und zu Fraunberg M. Molecular and biochemical studies of acute intermittent porphyria in 196 patients and their families. Clin Chem. 2002;48(11):1891-900.

8. Marsden JT, Rees DC. Urinary excretion of porphyrins, porphobilinogen and delta-aminolaevulinic acid following an attack of acute intermittent porphyria. J Clin Pathol. 2014;67(1):60-5.

9. Herrero C, Badenas C, Aguilera P, To-Figueras J. Acute intermittent porphyria: long-term follow up of 35 patients. Med Clin (Barc). 2015;145(8):332-7.

10. Balwani M, Wang B, Anderson KE, Bloomer JR, Bissell DM, Bonkovsky HL, et al. Acute hepatic porphyrias: recommendations for evaluation and long-term management. Hepatology. 2017;66(4):1314-22.

11. Stewart MF. Review of hepatocellular cancer, hypertension and renal impairment as late complications of acute porphyria and recommendations for patient follow-up. J Clin Pathol. 2012;65(11):976-80.

12. Pallet N, Mami I, Schmitt C, Karim Z, Francois A, Rabant M, et al. High prevalence of and potential mechanisms for chronic kidney disease in patients with acute intermittent porphyria. Kidney Int. 2015;88(2):386-95.

13. Baravelli CM, Sandberg S, Aarsand AK, Nilsen RM, Tollanes MC. Acute hepatic porphyria and cancer risk: a nationwide cohort study. J Intern Med. 2017;282(3):229-40.

14. Millward LM, Kelly P, Deacon A, Senior V, Peters TJ. Self-rated psychosocial consequences and quality of life in the acute porphyrias. I Inherit Metab Dis. 2001;24(7):733-47.

15. Bylesjo I, Wikberg A, Andersson C. Clinical aspects of acute intermittent porphyria in northern Sweden: a population-based study. Scand J Clin Lab Invest. 2009;69(5):612-8.

16. Naik H, Stoecker M, Sanderson SC, Balwani M, Desnick RJ. Experiences and concerns of patients with recurrent attacks of acute hepatic porphyria: a qualitative study. Mol Genet Metab. 2016;119(3):278-83.

17. Yang J, Zhu T, Zhao Y, Yu X, Zhu H, Jiang Y, et al. Acute intermittent porphyria in the North of China: the acute attack effect on quality of life and psychological condition. Biomed Res Int. 2018;2018:3216802.
18. Simon A, Pompilus F, Querbes W, Wei A, Strzok S, Penz C, et al. Patient perspective on acute intermittent porphyria with frequent attacks: a disease with intermittent and chronic manifestations. Patient. 2018;11(5):527-37.

19. Gouya L, Ventura P, Balwani M, Bissell DM, Rees DC, Stölzel U, et al. EXPLORE: a prospective, multinational, natural history study of patients with acute hepatic porphyria with recurrent attacks. Hepatology. 2020;71(5):1546-58

20. Jimenez-Monreal AM, Murcia MA, Gomez-Murcia V, Bibiloni Mdel M, Pons A, Tur JA, et al. Anthropometric and quality-of-life parameters in acute intermittent porphyria patients. Medicine. 2015;94(30):e1023.

21. Webster AC, Nagler EV, Morton RL, Masson P. Chronic kidney disease. Lancet. 2017;389(10075):1238-52.

22. Badia X, Roset M, Montserrat S, Herdman M, Segura A. The Spanish version of EuroQol: a description and its applications.\&nbsp;European Quality of Life scale. Med Clin (Barc). 1999;1 12(Suppl 1):79-85.

23. Hernandez G, Garin O, Pardo Y, Vilagut G, Pont À, Suárez M, et al. Validity of the EQ-5D-5L and reference norms for the Spanish population. Qual Life Res. 2018:27(9):2337-48.

24. Kauppinen R, Mustajoki P. Prognosis of acute porphyria: occurrence of acute attacks, precipitating factors, and associated diseases. Medicine. 1992;71(1):1-13.

25. Andersson C, Lithner F. Hypertension and renal disease in patients with acute intermittent porphyria. J Intern Med. 1994;236(2):169-75.

26. Menendez E, Delgado E, Fernandez-Vega F, Prieto MA, Bordiu E, Calle A, et al. Prevalence, diagnosis, treatment, and control of hypertension in Spain. Results of the diabetes study. Rev Esp Cardiol (Engl Ed). 2016;69(6):572-8

27. Sardh $E$, Wahlin $S$, Bjornstedt $M$, Harper $P$, Andersson DE. High risk of primary liver cancer in a cohort of 179 patients with acute hepatic porphyria. J Inherit Metab Dis. 2013;36(6):1063-71.

28. Saberi B, Naik H, Overbey JR, Erwin AL, Anderson KE, Bissell DM, et al. Hepatocellular carcinoma in acute hepatic porphyrias: results from the longitudinal study of the U.S. Porphyrias Consortium. Hepatology. 2020; https://doi.org/10.1002/hep.31460.

29. Floderus Y, Sardh E, Moller C, Andersson C, Rejkjaer L, Andersson DE, et al. Variations in porphobilinogen and 5-aminolevulinic acid concentrations in plasma and urine from asymptomatic carriers of the acute intermittent porphyria gene with increased porphyrin precursor excretion. Clin Chem. 2006;52(4):701-7.

\section{Publisher's note}

Springer Nature remains neutral with regard to jurisdictional claims in published maps and institutional affiliations.
Ready to submit your research? Choose BMC and benefit from:

- fast, convenient online submission

- thorough peer review by experienced researchers in your field

- rapid publication on acceptance

- support for research data, including large and complex data types

- gold Open Access which fosters wider collaboration and increased citations

- maximum visibility for your research: over $100 \mathrm{M}$ website views per year

At BMC, research is always in progress.

Learn more biomedcentral.com/submissions 